\section{OPEN ACCESS}

Edited by:

Andrea P. Rossi,

Integrated University Hospital Verona,

Reviewed by:

Valentina Muollo,

University of Verona, Italy

Georgia Colleluori,

Marche Polytechnic University, Italy

*Correspondence:

Alain Dagher

alain.dagher@mcgill.ca

Specialty section:

This article was submitted to

Obesity,

a section of the journa

Frontiers in Endocrinology

Received: 13 January 2021

Accepted: 10 March 2021

Published: 25 March 2021

Citation:

Morys F and Dagher A (2021) Poor

Metabolic Health Increases COVID-19-Related Mortality in the UK Biobank Sample.

Front. Endocrinol. 12:652765. doi: 10.3389/fendo.2021.652765

\title{
Poor Metabolic Health Increases COVID-19-Related Mortality in the UK Biobank Sample
}

Filip Morys and Alain Dagher*

Department of Neurology and Neurosurgery, Montreal Neurological Institute, McGill University, Montreal, QC, Canada

Previous studies link obesity and components of metabolic health, such as hypertension or inflammation, to increased hospitalizations and mortality of patients with COVID-19. Here, in two overlapping samples of over 1,000 individuals from the UK Biobank we investigate whether metabolic health as measured by waist circumference, dyslipidemia, hypertension, type 2 diabetes, and systemic inflammation is related to increased COVID19 infection and mortality rate. Using logistic regression and controlling for confounding variables such as socioeconomic status, age, sex or ethnicity, we find that individuals with worse metabolic health (measured on average eleven years prior to 2020) have an increased risk for COVID-19-related death (adjusted odds ratio: 1.75). We also find that specific factors contributing to increased mortality are increased serum glucose levels, systolic blood pressure and waist circumference.

Keywords: COVID-19, obesity, diabetes, hypertension, dyslipidemia

\section{INTRODUCTION}

Since the beginning of the COVID-19 pandemic, mounting evidence supports an association between obesity and poor outcomes (1-12). The association holds for both obesity and obesityassociated metabolic health - hypertension, diabetes, dyslipidemia, and systemic inflammation (13). Similarly, obesity and excess adipose tissue have also been associated with higher risk of SARS-CoV2 infection $(10,14,15)$.

Previous studies, however, have tended to use small sample sizes, focus predominantly on the effects of body mass index (BMI) as a measure of obesity, or not account for confounding factors, such as ethnicity or socioeconomic status $(14,16-18)$. Since ethnicity and socioeconomic status are themselves associated with obesity and metabolic health $(19,20)$, they could confound interpretation of analyses in patients with COVID-19.

Here, we aim to present a comprehensive evaluation of obesity-associated metabolic risk factors that might be related to poor health outcomes in SARS-CoV-2 infected patients while controlling for confounding variables and limiting potential collider bias, which has previously resulted in incorrect epidemiological conclusions (21-23). We investigate whether metabolic health is related to higher chance for SARS-CoV-2 infection, but also COVID-19-related death. 


\section{MATERIALS AND METHODS}

\section{Participants}

In this study, we used the UK Biobank dataset - a large scale study with extensive phenotyping carried out in the United Kingdom (24). This study was performed under UK Biobank application ID 35605. SARS-CoV-2 real-time PCR test results in the UK Biobank dataset are derived from the Public Health England microbiology database Second Generation Surveillance System that is dynamically linked to the UK Biobank database (25). Here we only included individuals who were recorded as tested for SARS-CoV-2. We distinguished between two samples for two aims of our project: Sample 1 - a larger sample $(\mathrm{n}=12,659)$ of all individuals who were tested for SARS-CoV-2 between $16^{\text {th }}$ March 2020 and $24^{\text {th }}$ August 2020, to investigate the risk of COVID-19 infection and how it is related to metabolic health; and Sample 2, a subset of Sample 1 consisting of individuals who tested positive for SARS-CoV-2 $(n=1,152)$. We also obtained data on mortality from COVID-19 for all the individuals included in our study population.
Sample characteristics can be found in Table 1. The discrepancies in number of patients who tested positive in both samples is due to outlier exclusions (see section: 'Measures used in the study'). Participants were classified as having metabolic syndrome based on the criteria in (26). All participants signed written informed consent prior to participating in the UK Biobank study, which was approved by the North-West Multicentre Research Ethics Committee (11/NW/0382). All UK Biobank actions are overseen by the UK Biobank Ethics Advisory Committee.

\section{Measures Used in the Study}

To investigate how metabolic health is related to SARS-CoV-2 infection and mortality rate, we used the following measures: waist circumference, serum triglyceride (TG), serum high density lipoprotein cholesterol (HDL), glycated hemoglobin (HbAlc), serum glucose (corrected for fasting times prior to blood drawing), serum C-reactive protein (27), previous type 2 diabetes diagnosis, resting systolic and diastolic blood pressure (mean of two measurements each), and hypertension diagnosis. In our analyses,

TABLE 1 | Participants characteristics.

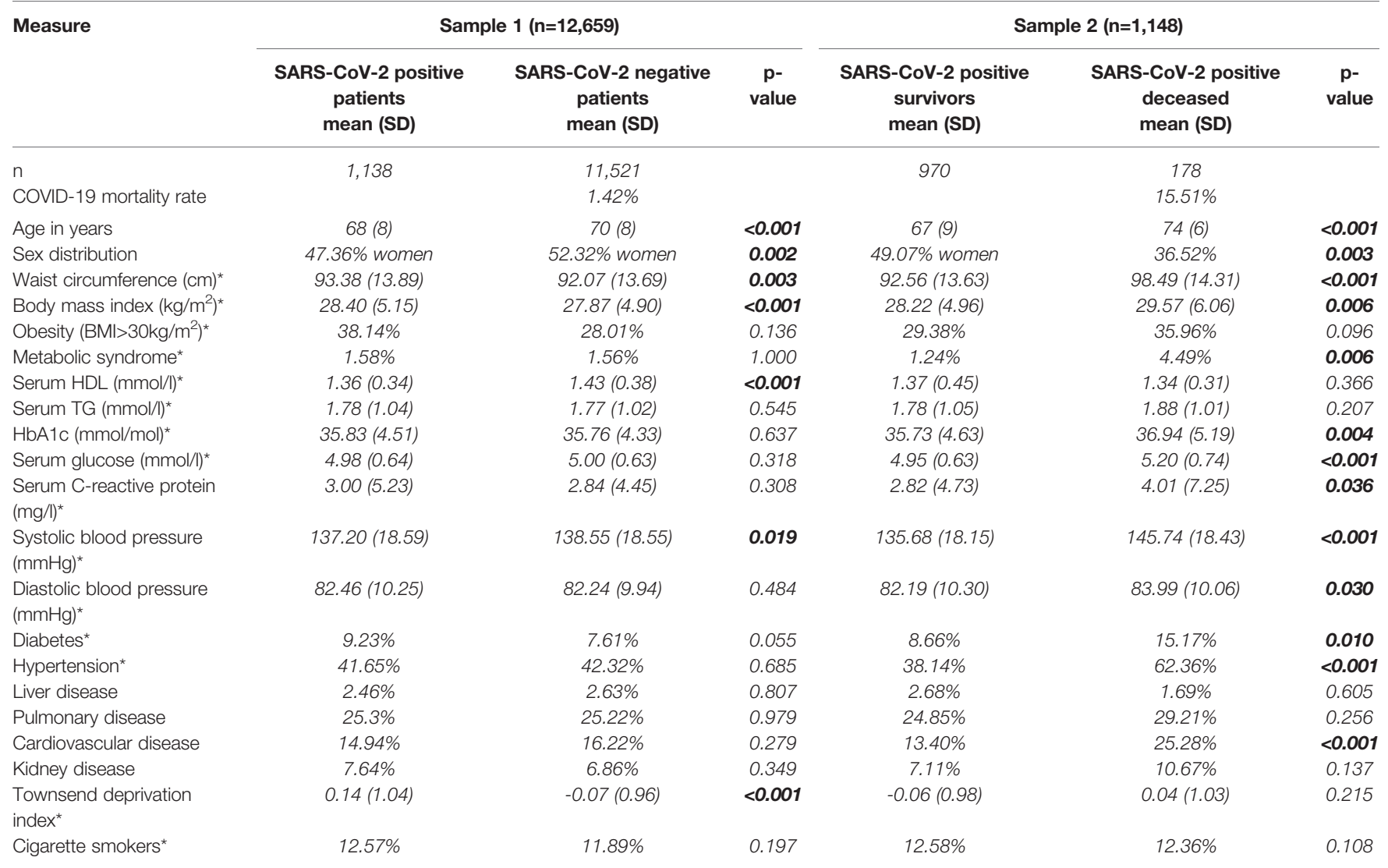

SD, standard deviation; BMI, body mass index; HDL, high density lipoprotein cholesterol; TG, triglycerides; HbA1C, hemoglobin A1c. Uncorrected p-values reflect significance of within samples differences calculated with t-tests in case of numerical variables and chi-squared tests in case of categorical variables.

Note that measures with an asterisk were obtained on average 11 years prior to 2020, while the other measures are from the time of testing.

Bold values indicate significant between-group differences. 
we also controlled for age, sex, socioeconomic status [Townsend deprivation index (28)], highest achieved educational qualifications, mean family income, smoking status, and ethnic background, the laboratory where COVID-19 testing was performed, and the origin of the sample used for COVID-19 test (e.g. nose, throat etc.). Based on the previous literature, we also controlled for past liver disease (ICD10 identifiers K7), cardiovascular disease (ICD10 identifiers I2 and I3), pulmonary disease (ICD10 identifiers J), and kidney disease (ICD10 identifiers N1) in all our analyses, as they are all related to the severity of COVID-19 (29-32). For Sample 2, we used mortality data provided by the UK Biobank - COVID-19-related death was described using the ICD10 identifier U07.1. All variables related to metabolic health and all confounding variables were collected on average 11 years prior to COVID-19 tests.

Prior to the analyses, all numeric variables were $\mathrm{z}$-scored, all serum level values were log-transformed, and we excluded outliers from the samples (2.2 interquartile range below 1st or above 3rd quartile). No other exclusions were applied. If participants were tested for SARS-CoV-2 more than once, they were considered positive if at least one test result was positive.

\section{Statistical Analyses}

The same analyses were performed for each of the two population samples. Data were analyzed using $\mathrm{R}$ (v. 3.6.0). First, using confirmatory factor analysis in lavaan (v. 0.6-7), we estimated the fit of a latent variable 'metabolic health', which consisted of waist circumference, serum C-reactive protein levels, a latent variable 'dyslipidemia' [serum HDL and TG levels; with loadings constrained to be equal between the two variables (33)], and two other latent variables, diabetes and hypertension. The latent variable 'diabetes' consisted of serum glucose and HbAlc levels, and type 2 diabetes diagnosis, while the latent variable 'hypertension' consisted of blood pressure measurements (systolic and diastolic blood pressure), and hypertension diagnosis (Figure 1). The model was estimated using robust maximum likelihood estimation and model's fit was evaluated using common indices: comparative fit index (CFI), root mean square error of approximation (RMSEA), and standardized root mean square residual (SRMR). Acceptable fit was defined as $\mathrm{CFI}>0.9$, RMSEA $<0.1$, and $\mathrm{SRMR}<0.08$.

Next, we extracted components of the latent variable 'metabolic health' for each participant and entered them in a logistic regression. The outcome variable in logistic regression for Sample 1 was COVID-19 test result, while for Sample 2 the outcome variable was COVID-19-related death.

In the analyses we used a set of confounding variables to calculate adjusted odds ratio: age, sex socioeconomic status, smoking status, ethnic background, test laboratory, and sample origin $(6,34-36)$.

Finally, for Sample 2 we explored how individual factors contributed to the COVID-19-related mortality by using a logistic regression with individual components of metabolic health, instead of the latent variable 'metabolic health'.

Overall, analyses using a latent variables approach allowed us to maximize information shared between different measured variables within the same metabolic domains, e.g. serum glucose levels, $\mathrm{HbA1C}$ levels and type 2 diabetes diagnosis for the latent variable 'diabetes'. Furthermore, adding continuous measures in this study, such as glucose levels or blood pressure, instead of only using type 2 diabetes or hypertension diagnosis, enabled us to use more information that is available in the dataset and investigate in depth how continuous metabolic health measures are related to SARS-CoV-2 infection and COVID-19 mortality.

A script for the analysis of the data as well as the output of statistical software can be found at https://github.com/ FilipMorys/COVID_MetS.

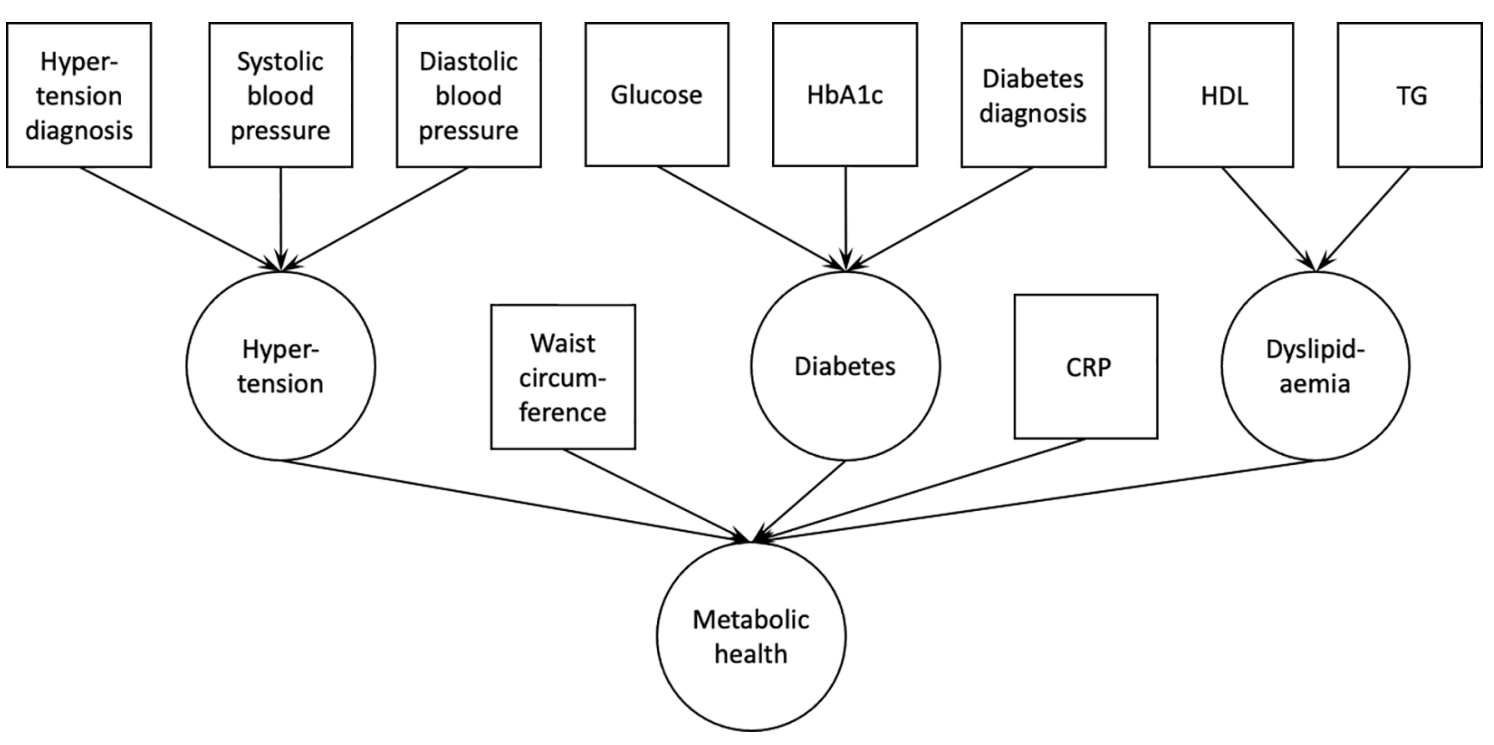

FIGURE 1 | Confirmatory factor analysis model used to derive the latent variable 'metabolic health'. Squares indicate measured variables; circles indicate latent variables. HbA1c, glycated hemoglobin A1c; HDL, high density lipoprotein cholesterol; TG, triglycerides; CRP, C-reactive protein. 


\section{RESULTS}

\section{Metabolic Health and the Risk of COVID-19 Infections}

In Sample 1, the confirmatory factor analysis provided an acceptable model fit $(\mathrm{CFI}=0.932$, RMSEA $=0.065$, SRMR $=0.040$ ). In the logistic regression the relation between metabolic health and the chance of having a positive SARS-CoV-2 test did not reach our pre-set statistical significance threshold $(\mathrm{p}=0.059$; odds ratio 1.10 ; 95\% confidence intervals (CI): 1.00-1.22).

\section{Metabolic Health Is Related to an Increased COVID-19-Related Mortality}

Among the individuals who tested positive, 178 people (16\%) died from COVID-19, allowing us to investigate how metabolic health influences COVID-19-related mortality. Here, the confirmatory factor analysis provided an acceptable model fit (CFI $=0.919$, RMSEA $=0.068$, SRMR $=0.049$ ). Logistic regression showed that obesity-associated metabolic impairment was related to an increased mortality rate among COVID-19 positive individuals $(\mathrm{p}<0.001)$ - adjusted odds ratio: 1.75 (95\% CI: $1.30-2.37$ ), pointing to a $75 \%$ increase for each unit increase on the metabolic health latent variable (Figure 2). Each unit increase on the metabolic health variable means increased serum glucose levels by $0.21 \mathrm{mmol} / \mathrm{l}, \mathrm{HbAlc}$ levels by $3.21 \mathrm{mmol} / \mathrm{mol}$, C-reactive protein levels by $2.18 \mathrm{mg} / \mathrm{l}$, triglyceride levels by 0.67 $\mathrm{mmol} / \mathrm{l}$, systolic blood pressure by $7.17 \mathrm{mmHg}$, diastolic blood pressure by $4.27 \mathrm{mmHg}$, waist circumference by $18.72 \mathrm{~cm}$, and decreased HDL cholesterol levels by $0.24 \mathrm{mmol} / \mathrm{l}$.

Exploratory analysis with individual components of metabolic health revealed that higher systolic blood pressure, higher serum glucose, and higher waist circumference increased the chance of COVID-19-related mortality - the adjusted odds ratio were 1.32 (95\% CI: 1.02-1.70), 1.27 (95\% CI: 1.04-1.55), and 1.34 (95\% CI:
1.05-1.72), respectively (systolic blood pressure: $\mathrm{p}=0.035$, glucose levels: $\mathrm{p}=0.020$, waist circumference: $\mathrm{p}=0.019$; Figure 2).

\section{DISCUSSION}

We investigated whether obesity-associated poor metabolic health, here defined by increased waist circumference, increased TG levels, decreased HDL levels, systemic inflammation, increased glucose and HbA1C levels, increased systolic and diastolic blood pressure, and the presence of type 2 diabetes and hypertension, is a risk factor for COVID-19 infection and mortality. We were able to show that a one unit increase of the latent variable 'metabolic health' results in a 75\% higher risk of death because of COVID-19. In contrast, the relation between metabolic health and the likelihood of test positivity was weaker, with an odds ratio of only 1.1 and a confidence interval that included the null effect. In sum, poor metabolic health contributes little to no risk of test positivity but substantially increases the odds of an adverse outcome. A strength of our analysis lies in the fact that the risk factor variables were measured prior to COVID-19 diagnosis and therefore were not influenced by the disease itself or possible treatment. Another strength is that we controlled for known shared risk factors between obesity and COVID-19 outcomes, such as socioeconomic status, ethnicity, and sex. We also used history of pulmonary, cardiovascular, kidney, and liver disease as covariates in our analyses, as these have been linked to COVID19 severity and mortality (29-32).

The results of our study are in line with previous reports linking obesity and individual components of metabolic health with poor COVID-19 outcomes and death $(1,5,7,10,12,18,37)$. In particular, the fact that waist circumference was related to COVID-19 mortality is consistent with a study showing that

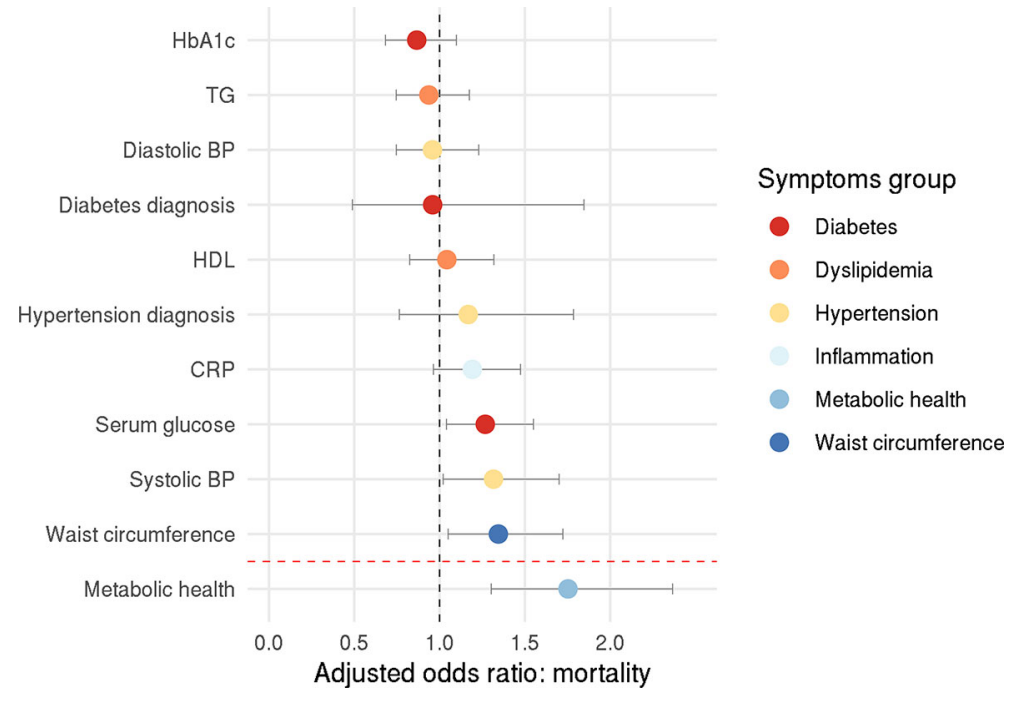

FIGURE 2 | Odds ratio for COVID-19-related death. Circles represent adjusted odds ratios, error bars represent 95\% confidence intervals. 
visceral adipose tissue is significantly related to the risk of admission to an intensive care unit in COVID-19 patients (38). Here, the authors argue that this might be in part due to increased local and systemic inflammation. In their review, Bansal et al. discuss some of the physiological mechanisms that might mediate the relationship between obesity, related comorbidities and worse outcomes of COVID-19 (39). These include an enhanced expression of the angiotensin converting enzyme 2 (ACE2), diabetes-related microvascular dysfunction, increased expression of pro-inflammatory cytokines, or activation of the renin-angiotensin-aldosterone system related to hypertension (39-41). Recent reports especially highlight the role of interleukin-6 (IL-6) in the pathogenesis of COVID-19, but also the role of IL- 6 inhibitors in COVID-19 therapy $(42,43)$. This is important given that adiposity is associated with chronic systemic inflammation, which generally delays immune response to pathogens and can also lead to worse outcomes in COVID-19 patients $(37,39,40,44)$. In general, our results suggest that a number of these mechanisms might also contribute to increased mortality from COVID-19.

Previous studies investigating COVID-19 in the UK Biobank used positive test results obtained between March $16^{\text {th }}$ and April $26^{\text {th }}$ as a proxy of severe COVID-19 $(18,45)$. The rationale for this is that, during this time, only patients admitted to hospitals and with COVID-19-like symptoms were tested for SARS-CoV-2. Here, we decided to not use this approach for several reasons. First, it is possible that individuals with COVID-19-like symptoms for which they were admitted to a hospital but who did not have COVID-19 were only infected after being admitted. In those cases, positive tests would not reflect COVID-19 severity. Second, it is not possible to determine the exact reason for which inpatients were tested for SARS-CoV-2; positive test results might therefore not only reflect severity of COVID-19 disease, but also testing in anticipation of isolating patients admitted for other reasons. For example, patients having to undergo unrelated medical procedures might have received precautionary testing. We therefore recommend that studies that used this approach as a proxy for COVID-19 severity be interpreted with caution.

For the interpretation of our and similar results from the UK Biobank, it is important to note that the UK Biobank is not a sample representative of the entire UK population and therefore the findings might not be generalizable (46). Infection and mortality rates calculated here should not be used as an indicator of true prevalence and mortality rate in the general population. Furthermore, observational studies such as ours are prone to collider bias, which has already been identified in UK Biobank COVID-19 investigations (23). Collider bias occurs when the sample population is conditioned on a variable that correlates with the variables of interest. For example, during the time period of the current study, it is thought that health workers were more likely to be tested for SARS-CoV-2, which may have contributed to incorrect conclusion that cigarette smoking is protective, as health care workers have a lower incidence of smoking (23). Our study population includes individuals who were seen in a health care setting, who may therefore have a higher incidence of obesity and poorer metabolic health than the general population. However, it is difficult to see how this would account for the effect of obesity on death from COVID-19 among people who tested positive for the virus.

In addition, COVID-19 testing in the UK during the time period of this study was generally restricted to individuals with symptoms such as fever, cough, or loss of smell or taste asymptomatic COVID-19 individuals were less likely to be tested. This might further increase the extent of collider bias in this and similar studies investigating the predictors of COVID19 severity or mortality. Current strategies to account for such bias or measure the extent thereof rely on models and strong assumptions that might be incorrect. We therefore suggest that our findings be interpreted with caution. Nonetheless, our model did account for potential collider variables or confounds such as socioeconomic status, ethnicity and age.

Finally, in this study we only used limited data pertaining to COVID-19 diagnosis or mortality. Information on the seriousness of illness, treatment administered, or actual cause of death were not available.

In sum, we used the UK Biobank dataset to confirm that, in individuals who tested positive for COVID-19 in the early stages of the pandemic, metabolic health, and especially visceral adiposity, hypertension, and serum glucose levels, were associated with an increased risk of death.

\section{DATA AVAILABILITY STATEMENT}

The data analyzed in this study is subject to the following licenses/restrictions: The dataset can be obtained from the UK Biobank. Requests to access these datasets should be directed to https://www.ukbiobank.ac.uk.

\section{ETHICS STATEMENT}

The studies involving human participants were reviewed and approved by North-West Multi-centre Research Ethics Committee. The patients/participants provided their written informed consent to participate in this study.

\section{AUTHOR CONTRIBUTIONS}

Both authors contributed equally to all stages of the research. All authors contributed to the article and approved the submitted version.

\section{FUNDING}

This work was supported by a Foundation Scheme award to AD from the Canadian Institutes of Health Research. The funders had no role in study design, data collection and analysis, decision to publish, or preparation of the manuscript. 


\section{REFERENCES}

1. Dietz W, Santos-Burgoa C. Obesity and its Implications for COVID-19 Mortality. Obesity (2020) 28:1005. doi: 10.1002/oby.22818

2. Stefan N, Birkenfeld AL, Schulze MB, Ludwig DS. Obesity and impaired metabolic health in patients with COVID-19. Nat Rev Endocrinol (2020) 16:341-2. doi: 10.1038/s41574-020-0364-6

3. Deng L, Zhang J, Wang M, Chen L. Obesity is associated with severe COVID19 but not death: a dose-response meta-analysis. Epidemiol Infect (2021) 1-27. doi: 10.1017/S0950268820003179

4. Huang Y, Lu Y, Huang YM, Wang M, Ling W, Sui Y, et al. Obesity in patients with COVID-19: a systematic review and meta-analysis. Metabolism (2020) 113:154378. doi: 10.1016/j.metabol.2020.154378

5. Gao F, Zheng KI, Wang XB, Sun QF, Pan KH, Wang TY, et al. Obesity Is a Risk Factor for Greater COVID-19 Severity. Diabetes Care (2020) 43:E72-4. doi: $10.2337 / \mathrm{dc} 20-0682$

6. Zhu Z, Hasegawa K, Ma B, Fujiogi M, Camargo CA, Liang L. Association of obesity and its genetic predisposition with the risk of severe COVID-19: Analysis of population-based cohort data. Metabolism (2020) 112:154345. doi: 10.1016/j.metabol.2020.154345

7. Zhao X, Gang X, He G, Li Z, Lv Y, Han Q, et al. Obesity Increases the Severity and Mortality of Influenza and COVID-19: A Systematic Review and MetaAnalysis. Front Endocrinol (Lausanne) (2020) 11:595109. doi: 10.3389/ fendo.2020.595109

8. Martos-Benítez FD, Soler-Morejón CD, García-del Barco D. Chronic comorbidities and clinical outcomes in patients with and without COVID-19: a large population-based study using national administrative healthcare open data of Mexico. Intern Emerg Med (2021), 1. doi: 10.1007/s11739-020-02597-5

9. Breland JY, Wong MS, Steers WN, Yuan AH, Haderlein TP, Washington DL. Body Mass Index and Risk for Severe COVID-19 among Veterans Health Administration Patients. Obesity (2021). doi: 10.1002/oby.23121. oby.23121.

10. Jayawardena R, Jeyakumar DT, Misra A, Hills AP, Ranasinghe P. Obesity: A potential risk factor for infection and mortality in the current COVID-19 epidemic. Diabetes Metab Syndr Clin Res Rev (2020) 14:2199-203. doi: 10.1016/j.dsx.2020.11.001

11. Chiappetta S, Sharma AM, Bottino V, Stier C. COVID-19 and the role of chronic inflammation in patients with obesity. Int J Obes (2020) 44:1790-2. doi: 10.1038/s41366-020-0597-4

12. Peña JE, Rascón-Pacheco RA, Ascencio-Montiel IJ, González-Figueroa E, Fernández-Gárate JE, Medina-Gómez OS, et al. Hypertension, Diabetes and Obesity, Major Risk Factors for Death in Patients With COVID-19 in Mexico. Arch Med Res (2020) 16:S0188-4409(20):32243-8. doi: 10.1016/ j.arcmed.2020.12.002

13. Alberti KG, Eckel RH, Grundy SM, Zimmet PZ, Cleeman JI, Donato KA, et al. Harmonizing the metabolic syndrome: a joint interim statement of the International Diabetes Federation Task Force on Epidemiology and Prevention; National Heart, Lung, and Blood Institute; American Heart Association; World Heart Federation; International. Circulation (2009) 120:1640-5. doi: 10.1161/CIRCULATIONAHA.109.192644

14. Yates T, Razieh C, Zaccardi F, Davies MJ, Khunti K. Obesity and risk of COVID-19: analysis of UK biobank. Prim Care Diabetes (2020) 14(5):566-7. doi: 10.1016/j.pcd.2020.05.011

15. de Frel DL, Atsma DE, Pijl H, Seidell JC, Leenen PJM, Dik WA, et al. The Impact of Obesity and Lifestyle on the Immune System and Susceptibility to Infections Such as COVID-19. Front Nutr (2020) 7:597600. doi: 10.3389/ fnut.2020.597600

16. Townsend MJ, Kyle TK, Stanford FC. Outcomes of COVID-19: disparities in obesity and by ethnicity/race. Int J Obes (2020) 44:1807-9. doi: 10.1038/ s41366-020-0635-2

17. Xie J, Zu Y, Alkhatib A, Pham TT, Gill F, Jang A, et al. Metabolic Syndrome and COVID-19 Mortality Among Adult Black Patients in New Orleans. Diabetes Care (2020) 44(1):188-93. doi: 10.2337/dc20-1714

18. Hamer M, Gale CR, Kivimäki M, Batty GD. Overweight, obesity, and risk of hospitalization for COVID-19: A community-based cohort study of adults in the United Kingdom. Proc Natl Acad Sci (2020) 117(35):21011-13. doi: $10.1073 /$ pnas.2011086117

19. Arroyo-Johnson C, Mincey KD. Obesity Epidemiology Worldwide. Gastroenterol Clin North Am (2016) 45:571-9. doi: 10.1016/j.gtc.2016.07.012
20. Pigeyre M, Rousseaux J, Trouiller P, Dumont J, Goumidi L, Bonte D, et al How obesity relates to socio-economic status: Identification of eating behavior mediators. Int J Obes (2016) 40:1794-801. doi: 10.1038/ijo.2016.109

21. Gaibazzi N, Tuttolomondo D, Guidorossi A, Botti A, Tedeschi A, Martini C, et al. Smoking Prevalence is Low in Symptomatic Patients Admitted for COVID-19. Cold Spring Harb Lab Press (2020). doi: 10.1101/ 2020.05.05.20092015

22. Miyara M, Tubach F, POURCHER V, Morelot-Panzini C, Pernet J, Haroche J, et al. Low rate of daily active tobacco smoking in patients with symptomatic COVID-19. Qeios (2020). doi: 10.32388/wpp19w.4

23. Griffith GJ, Morris TT, Tudball MJ, Herbert A, Mancano G, Pike L, et al. Collider bias undermines our understanding of COVID-19 disease risk and severity. Nat Commun (2020) 11:5749. doi: 10.1038/s41467-020-19478-2

24. Sudlow C, Gallacher J, Allen N, Beral V, Burton P, Danesh J, et al. UK Biobank: An Open Access Resource for Identifying the Causes of a Wide Range of Complex Diseases of Middle and Old Age. PloS Med (2015) 12: e1001779. doi: 10.1371/journal.pmed.1001779

25. Armstrong J, Rudkin JK, Allen N, Crook DW, Wilson DJ, Wyllie DH, et al. Dynamic linkage of covid-19 test results between public health england's second generation surveillance system and uk biobank. Microb Genomics (2020) 6:1-9. doi: 10.1099/mgen.0.000397

26. Alberti KGMM, Eckel RH, Grundy SM, Zimmet PZ, Cleeman JI, Donato KA, et al. Harmonizing the metabolic syndrome: A joint interim statement of the international diabetes federation task force on epidemiology and prevention; National heart, lung, and blood institute; American heart association; World heart federation; International atherosclerosis society; And international association for the study of obesity. Circulation (2009) 120:1640-5. doi: 10.1161/CIRCULATIONAHA.109.192644

27. Elliott P, Peakman TCUK Biobank. The UK Biobank sample handling and storage protocol for the collection, processing and archiving of human blood and urine. Int J Epidemiol (2008) 37:234-44. doi: 10.1093/ije/dym276

28. Townsend $\mathrm{P}$, Phillimore $\mathrm{P}$, Beattie A. Health and Deprivation: Inequality and the North. London: Croom Helm (1988). Available at: https://books.google.ca/ books?id=K9kOAAAAQAAJ.

29. Ghoneim S, Butt MU, Hamid O, Shah A, Asaad I. The incidence of COVID19 in patients with metabolic syndrome and non-alcoholic steatohepatitis: A population-based study. Metab Open (2020) 8:100057. doi: 10.1016/ j.metop.2020.100057

30. Olloquequi J. COVID-19 Susceptibility in chronic obstructive pulmonary disease. Eur J Clin Invest (2020) 50:e13382. doi: 10.1111/eci.13382

31. Henry BM, Lippi G. Chronic kidney disease is associated with severe coronavirus disease 2019 (COVID-19) infection. Int Urol Nephrol (2020) 52:1193-4. doi: 10.1007/s11255-020-02451-9

32. Bansal M. Cardiovascular disease and COVID-19. Diabetes Metab Syndr Clin Res Rev (2020) 14:247-50. doi: 10.1016/j.dsx.2020.03.013

33. Little TD, Lindenberger U, Nesselroade JR. On selecting indicators for multivariate measurement and modeling with latent variables: When "good" indicators are bad and "bad" indicators are good. Psychol Methods (1999) 4:192-211. doi: 10.1037/1082-989X.4.2.192

34. Wu Z, McGoogan JM. Characteristics of and Important Lessons from the Coronavirus Disease 2019 (COVID-19) Outbreak in China: Summary of a Report of 72314 Cases from the Chinese Center for Disease Control and Prevention. JAMA - J Am Med Assoc (2020) 323:1239-42. doi: 10.1001/ jama.2020.2648

35. Goyal P, Choi JJ, Pinheiro LC, Schenck EJ, Chen R, Jabri A, et al. Factors associated with hospital admission and critical illness among 5279 people with coronavirus disease 2019 in New York City: prospective cohort study. BMJ (2020) 369:m1966. doi: 10.1136/bmj.m1966

36. Petrilli CM, Jones SA, Yang J, Rajagopalan H, O’Donnell L, Chernyak Y, et al. Factors associated with hospital admission and critical illness among 5279 people with coronavirus disease 2019 in New York City: Prospective cohort study. BMJ (2020) 369:m1966. doi: 10.1136/bmj.m1966

37. Costa FF, Rosário WR, Ribeiro Farias AC, de Souza RG, Duarte Gondim RS, Barroso WA. Metabolic syndrome and COVID-19: An update on the associated comorbidities and proposed therapies. Diabetes Metab Syndr Clin Res Rev (2020) 14:809-14. doi: 10.1016/j.dsx.2020.06.016

38. Battisti S, Pedone C, Napoli N, Russo E, Agnoletti V, Nigra SG, et al. Computed tomography highlights increased visceral adiposity associated 
with critical illness in covid-19. Diabetes Care (2020) 43:e129-30. doi: $10.2337 / \mathrm{dc} 20-1333$

39. Bansal R, Gubbi S, Muniyappa R. Metabolic Syndrome and COVID 19: Endocrine-Immune-Vascular Interactions Shapes Clinical Course. Endocrinology (2020) 161:1-15. doi: 10.1210/endocr/bqaa112

40. Mohammad S, Aziz R, Al Mahri S, Malik SS, Haji E, Khan AH, et al. Obesity and COVID-19: what makes obese host so vulnerable? Immun Ageing (2021) 18:1-10. doi: 10.1186/s12979-020-00212-x

41. Abu-Farha M, Al-Mulla F, Thanaraj TA, Kavalakatt S, Ali H, Abdul Ghani M, et al. Impact of Diabetes in Patients Diagnosed With COVID-19. Front Immunol (2020) 11:576818. doi: 10.3389/fimmu.2020.576818

42. Nasonov E, Samsonov M. The role of Interleukin 6 inhibitors in therapy of severe COVID-19. BioMed Pharmacother (2020) 131:110698. doi: 10.1016/ j.biopha.2020.110698

43. Sinha P, Mostaghim A, Bielick CG, McLaughlin A, Hamer DH, Wetzler LM, et al. Early administration of interleukin-6 inhibitors for patients with severe COVID-19 disease is associated with decreased intubation, reduced mortality, and increased discharge. Int J Infect Dis (2020) 99:28-33. doi: 10.1016/ j.ijid.2020.07.023

44. Morys F, Dadar M, Dagher A. Association between mid-life obesity, its metabolic consequences, cerebrovascular disease and cognitive decline. J Clin Endocrinol Metab (2021) 2:dgab135. doi: 10.1210/clinem/ dgab135

45. Kuo C-L, Pilling LC, Atkins JL, Masoli JAH, Delgado J, Kuchel GA, et al. APOE e4 Genotype Predicts Severe COVID-19 in the UK Biobank Community Cohort. J Gerontol Ser A (2020) XX:1-2. doi: 10.1093/gerona/ glaa131

46. Fry A, Littlejohns TJ, Sudlow C, Doherty N, Adamska L, Sprosen T, et al. Comparison of Sociodemographic and Health-Related Characteristics of UK Biobank Participants with Those of the General Population. Am J Epidemiol (2017) 186:1026-34. doi: 10.1093/aje/kwx246

Conflict of Interest: The authors declare that the research was conducted in the absence of any commercial or financial relationships that could be construed as a potential conflict of interest.

Copyright $\odot 2021$ Morys and Dagher. This is an open-access article distributed under the terms of the Creative Commons Attribution License (CC BY). The use, distribution or reproduction in other forums is permitted, provided the original author(s) and the copyright owner(s) are credited and that the original publication in this journal is cited, in accordance with accepted academic practice. No use, distribution or reproduction is permitted which does not comply with these terms. 